\title{
PLANT SPECIE CLASSIFICATION USING SINUOSITY COEFFICIENTS OF LEAVES
}

\author{
JULES R. KALA ${ }^{凶}$, AND SERESTINA VIRIRI \\ School of Mathematics, Statistics and Computer Science \\ University of KwaZulu-Natal, Durban, 4000, South_Africa \\ e-mail: raymondkala1@gmail.com; viriris@ukzn.ac.za \\ (Received August 11, 2017; revised March 12, 2018; accepted March 12, 2018)
}

\begin{abstract}
Forests are the lungs of our planet. Conserving the plants may require the development of an automated system that will identify plants using leaf features such as shape, color, and texture. In this paper, a leaf shape descriptor based on sinuosity coefficients is proposed. The sinuosity coefficients are defined using the sinuosity measure, which is a measure expressing the degree of meandering of a curve. The initial empirical experiments performed on the LeafSnap dataset on the usage of four sinuosity coefficients to characterize the leaf images using the Radial Basis Function Neural Network (RBF) and Multilayer Perceptron (MLP) classifiers achieved accurate classification rates of $88 \%$ and $65 \%$, respectively. The proposed feature extraction technique is further enhanced through the addition of leaf geometrical features, and the accurate classification rates of $93 \%$ and $82 \%$ were achieved using RBF and MLP, respectively. The overall results achieved showed that the proposed feature extraction technique based on the sinuosity coefficients of leaves, complemented with geometrical features improve the accuracy rate of plant classification using leaf recognition.
\end{abstract}

Keywords: leaf recognition, plant classification, sinuosity coefficients, sinuosity measure.

\section{INTRODUCTION}

Plants are the key elements of life on earth: plants are source of food for animals, insects, and are source of energy, and they also help in the process of climate control. The leaves are one of the most visible and vital parts of a plant. They have valuable information about plant environment and can help identify the species to which a plant belongs to (von Linné, 1788). Botanists use information from leaves such as the tooth pattern on the leaf margin to classify plants (Partington, 1837).

The sinuosity of a given curve represents the degree of meandering of that curve. It has been extensively used in the domain of medical science for the analysis of the meandering of a spinal column (Tanguy and Peuchot, 2002) and in hydrography to evaluate the degree of meandering of a river (Langbein and Leopold, 1966). This article is an extension of a paper presented by Kala et al. (2016), where the sinuosity coefficients were used to characterize leaf shapes. In this article, a leaf image is divided into four sections and the sinuosity coefficients are computed from each section to construct a model for leaf classification.

To demonstrate the accuracy of the proposed model, the LeafSnap data set of more than 1840 leaf images of 184 species is used. The initial experiment is used to evaluate the efficacy of the sinuosity coefficients to characterize a leaf shape. The second experiment is designed to assess if combining the sinuosity coefficients to other geometrical features such as rectangularity, circularity, sphericity and aspect ratio will improve the classification rate obtained in the first experiment.

\section{RELATED WORKS}

Studies on plant classification using leaves have been carried out by several researchers with promising results. In the following subsections, some related works on plant classification using leaf images and the use of the sinuosity measure are presented.

\section{PLANT CLASSIFICATION USING LEAVES}

Kalyoncu and Toygar (2015) proposed a method for plant recognition using leaf images based on the combination of new and well know feature extraction techniques and classification algorithms. The proposed method is based on the Linear Discriminant Classifier to classify extracted features. The proposed method achieved a classification rate of $95 \%$ on the Flavia dataset and $70 \%$ on the Leafsnap dataset with the Local Discriminant Classifier (LDC) as a classifier. 
Lei et al. (2014), derived two orthogonal locally discriminant spline embedding techniques (OLDSEI and OLDSE-II) from a Local Spline embedding (LSE) and a Maximum Margin Criterion (MMC). The plant leaves are mapped into a leaf subspace in order to identify the essential leaf manifold. The proposed approach was applied to a subset of the ICL leaf image database containing 480 leaf images of 16 different species. It achieved an average classification rate of $95 \%$. The proposed method was also applied to the Swedish leaf database and achieved an average classification rate of $90 \%$.

Cerutti et al. (2014) presented a method for the analysis of the spatial properties of teeth on the leaf margin using structured representation. They proposed a sequential representation of leaf margins where the teeth are viewed as symbol of a multivariate real value alphabet. They applied the proposed technique to design a mobile application for tree identification. The proposed method achieved an average classification rate of $90 \%$ on leaf images from the Pl@ntLeaves database.

Yanikoglu et al. (2014) developed a system for plant identification using leaf images. The proposed method is based on the combination of shape, color, and texture features to overcome the difficulties encountered during the identification process. The proposed system achieved a classification rate of $81 \%$ on Image-CLEF' 12 leaf image dataset with Support Vector Machine as a classifier.

Wilf et al. (2016) combined shape features to venation features to construct a system for plant classification using leaf images. The proposed method requires a chemical pre-treatment of the leaf to magnify the venation on the leaf before taking the image. The proposed method cannot be easily automated because of the chemical process needed to expose the venation.

$\mathrm{Fu}$ et al. (2004) proposed an ontology based leaf classification system using leaf shape combined with leaf venation. The proposed system contained an algorithm for shape analysis and a neural network classifier to organize the leaves according to their tooth pattern. There was a $94 \%$ accuracy rate in recognition of tooth patterns of a set of three plant species.

\section{APPLICATION OF SINUOSITY MEASURE}

The sinuosity measure has been applied in many fields of science, such as Geography, Biology and Medical Science as a parameter to explain other natural phenomena such as the meandering of a river, etc.
In Langbein and Leopold (1966), the sinuosity measure was used to evaluate the degree of meandering of a river. It was also explained that the meandering in the case of a river is the result of an erosion process tending toward the most stable form in which the variability of certain essential properties such as velocity and depth are minimized.

Jaekel and Wake (2007) presented an application of the sinuosity measure for the characterization of the webbing on a salamander foot to demonstrate the morphological changes performed on the animal foot to adapt to a given surface.

Tanguy and Peuchot (2002) developed a method for the analysis of spine meandering for the early detection of spine deformation. Furthermore, Lazarus and Constantine (2013) used the sinuosity measure to demonstrate that there were rivers on Mars surface by analyzing the planet surface to detect deep meandering shapes.

\section{MATERIALS AND METHOD}

The proposed model for plant classification using leaf recognition is depicted in Fig. 1. The stages of the model are mainly preprocessing, feature extraction and classification. The output of the model is the plant specie or class to which the input leaf image belongs to.

Thresholding phase on this process is the simplest segmentation technique used to create a binary representation of an input image, using Eq. 1.

$$
f(x, y)= \begin{cases}0 & \text { if } f(x, y)>T, \\ 255 & \text { if } f(x, y) \leq T .\end{cases}
$$

The difficulty with using the thresholding method is determining the threshold $(T)$ which depends on the input image.

For the edges detection purpose, Sobel operator is used, because it can detect edges and their orientation (Gonzalez and Woods, 1992). A Sobel operator is obtained from the Prewit operator by increasing the weight on the central coefficients. Sobel edges detection is implemented using the masks in Fig. 2.

\section{DATABASE SAMPLING}

The sampling method used in our experiment is based on the formula extracted from (Naing et al., 2006), to create a sample from a finite population as defined in Eq. 2,

$$
n^{\prime}=\frac{N Z^{2} P(1-P)}{d^{2}(N-1)+Z^{2} P(1-P)},
$$




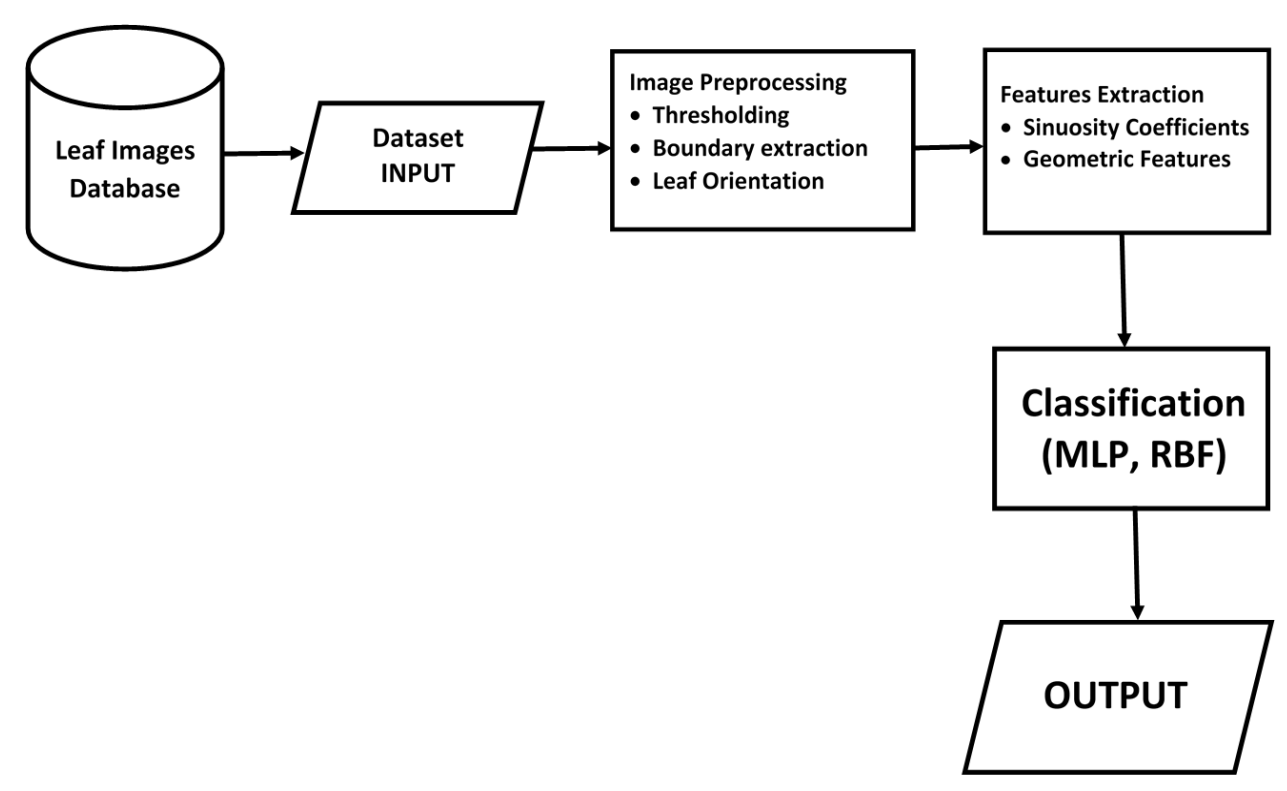

Fig. 1. Proposed model for plant classification

where $n^{\prime}$ is the sample size with the finite population; $N$ is the population size; $Z$ is the $\mathrm{Z}$ statistic for a level of confidence which is usually equal to $1.96 ; P$ is the expect proportion; $d$ is the precision equal to 0.05 . For the LeafSnap dataset the minimum sample obtained using the formula is equal to 336 leaf images.

\section{DATASET}

This study was conducted using LeafSnap created by Kumar et al. (2012), which is a leaf image database that contains 23147 Lab images and 7719 Field images of 185 tree species. For all the experiments 1800 leaves of 100 species were randomly selected from the LeafSnap database, using the sampling approach defined in Eq. 2 by considering only the field leaf images with uniform background to avoid additional computation cost. Fig. 3 shows some of the randomly selected leaf images.

\section{IMAGE PREPROCESSING}

Let $M$ be a leaf color image, the features for the characterization of $M$ will be extracted from the image greyscale. Let $I$ be the binary representation of the greyscale components of $M$. Considering a set of boundary points $\left(x_{i}, y_{i}\right)_{i=1, \ldots, n}$ of the binary image. The following elements are used for the construction of the Minimum Bounding Rectangle (MBR) in (Kala et al., 2014; Chaudhuri and Samal, 2007) will be considered:

\begin{tabular}{|c|c|c|}
\hline$\frac{-1}{\sqrt{12}}$ & $\frac{-1}{\sqrt{3}}$ & $\frac{-1}{\sqrt{12}}$ \\
\hline 0 & 0 & 0 \\
\hline$\frac{1}{\sqrt{12}}$ & $\frac{1}{\sqrt{3}}$ & $\frac{1}{\sqrt{12}}$ \\
\hline
\end{tabular}

\begin{tabular}{|l|l|l|}
\hline$\frac{-1}{\sqrt{12}}$ & 0 & $\frac{1}{\sqrt{12}}$ \\
\hline$\frac{-1}{\sqrt{3}}$ & 0 & $\frac{1}{\sqrt{3}}$ \\
\hline$\frac{-1}{\sqrt{12}}$ & 0 & $\frac{1}{\sqrt{12}}$ \\
\hline
\end{tabular}

Fig. $2.3 \times 3$ Sobel masks.

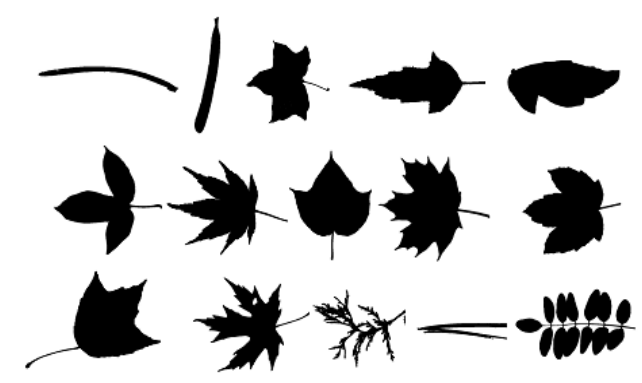

Fig. 3. Sample of the randomly selected leaf images from LeafSnap

Determination of the boundary centroid. The centroid, $(\bar{x}, \bar{y})$, is the average of the coordinates of the boundary points, defined as:

$$
(\bar{x}, \bar{y})=\left(\frac{1}{n} \sum_{i=1}^{n} x_{i}, \frac{1}{n} \sum_{i=1}^{n} y_{i}\right) .
$$

Determination of the image orientation and the image principal axes. The least square method applied to the coordinates of the boundary points will be used to determine the orientation of the image shape and the main axis dividing the object shape into 4 parts. 
The formula given in Eq. 4 is used to obtain the object orientation, $\theta$ (Chaudhuri and Samal, 2007; Kala et al., 2014).

$$
\tan 2 \theta=\frac{2 \sum_{i=1}^{n}\left(x_{i}-\bar{x}\right)\left(y_{i}-\bar{y}\right)}{\sum_{i=1}^{n}\left[\left(x_{i}-\bar{x}\right)^{2}-\left(y_{i}-\bar{y}\right)^{2}\right]} .
$$

This formula is obtained by using the Eq. 5 of the line passing through the centroid with the angle $\theta$,

$$
x \tan \theta-y+\bar{y}-\bar{x} \tan \theta=0,
$$

and the perpendicular distance from that line to an edge point which is defined as:

$$
p_{i}=\left(x_{i}-\bar{x}\right) \sin \theta-\left(y_{i}-\bar{y}\right) \cos \theta .
$$

The value of the angle $\theta$ defined in Eq. 4 is computed by minimizing $P$ defined in Eq. 7 with respect to $\theta$ $(\partial P / \partial \theta=0)$

$$
P=\sum_{i=1}^{n}\left[\left(x_{i}-\bar{x}\right) \sin \theta-\left(y_{i}-\bar{y}\right) \cos \theta\right]^{2} .
$$

\section{Sinuosity measure}

Let $\left(x_{i}, y_{i}\right), i=1,2, \ldots, n$, the coordinates of points composing the curve $l$. If $l$ is a continuously derivable curve having at least one inflexion point, then the sinuosity of $l$ is equal to the ratio between the length of $l$ and the length of the straight line joining the two end points $A\left(x_{0}, y_{0}\right)$ and $B\left(x_{n}, y_{n}\right)$ of $l$. The sinuosity measure of the curve $l$ is expressed by the following equation:

$$
S_{l}=\frac{\sum_{i=1}^{n} \sqrt{\left(x_{i}-x_{i-1}\right)^{2}+\left(y_{i}-y_{i-1}\right)^{2}}}{\sqrt{\left(x_{n}-x_{0}\right)^{2}+\left(y_{n}-y_{0}\right)^{2}}} .
$$

The values generated by Eq. 8 are from 1 (for a straight line) to infinity (closed loop where the shortest path length is zero) or for an infinitely long curve (Lazarus and Constantine, 2013). Lets consider a curve formed by two inverted semicircles located in the same plane. The sinuosity measure of this curve is equal to: $S=$ $\frac{\pi}{2} \approx 1.5708$. In order to evaluate the sinuosity measure of a curve $C$, one should make sure that $C$ is continuous between its two ends. Generally the sinuosity measure is evaluated in dimension two but it is also valid in dimension three (Tanguy and Peuchot, 2002). The basic classification of the sinuosity is either Strong: $1 \ll S$ or Weak: $S \approx 1$ ). This basic classification is the point of interest of this paper because the sinuosity measure will provide the information needed for the classification of the leaf edge into two groups (smooth leaf="Weak" edge and jagged leaf="Strong" edge).

\section{Sinuosity coefficients}

The sinuosity measure of a complete leaf boundary is infinite because leaf shape is a close contour. In order to apply the sinuosity measure to a leaf contour, a leaf shape is divided into four or more different parts as shown in Fig. 4. In order to obtain the leaf shape sinuosity coefficients, the sinuosity measure of each of the following curves $(U V, U H),(U V, L H),(L V, U H)$ and $(L V, L H)(U V$ : upper vertical point, $U H$ : upper horizontal point, $L V$ : Lower Vertical point and $L H$ : Lower horizontal point) was evaluated using Eq. 8. The sinuosity coefficients of a leaf shape is a vector of sorted values of the sinuosity measure of each curves composing the leaf shape. Fig. 5 shows some leaves with their associated sinuosity coefficients.

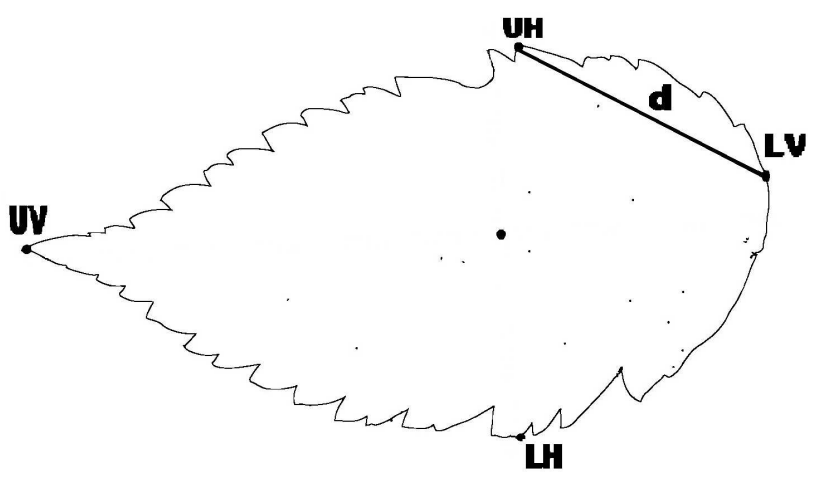

Fig. 4. Leaf shape information: LH: Lower Horizontal point, UH: Upper Horizontal point, UV: Upper Vertical point, LV: Lower Vertical point, d:distance between $U H$ and $L V$.

\section{GEOMETRIC FEATURES}

The rectangularity $(R)$ represents the ratio between the leaf area $\left(A_{\text {leaf }}\right)$ and the area of the minimum bounding rectangle. It evaluate how the leaf shape is close to a rectangle shape,

$$
R=\frac{A_{\text {leaf }}}{D_{\max } \times D_{\min }}
$$

The aspect ratio $(A)$ is the ratio between the maximum length $\left(D_{\max }\right)$ and the minimum length $\left(D_{\min }\right)$ of the minimum bounding rectangle,

$$
A=\frac{D_{\max }}{D_{\min }} .
$$

The sphericity $(S)$ is expressed by the following equation:

$$
S=\frac{r_{i}}{r_{c}}
$$

where: 
(a)

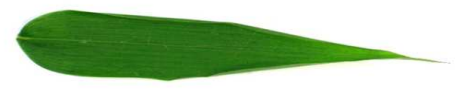

$(1.34,1.40,1.43,1.52)$

(c)

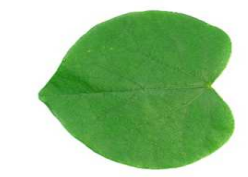

$(1.45,1.58,1.69,2.04)$ (b)

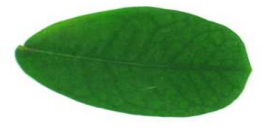

$(1.34,1.40,1.43,1.52)$

(d)

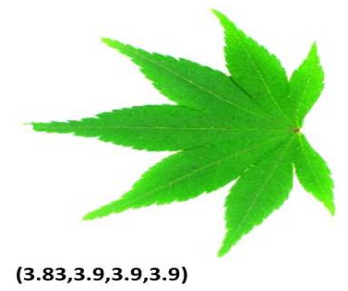

Fig. 5. Sinuosity coefficients of four leaf shapes: the sinuosity coefficients of the curves (UV;UH), (UV;LH), $(L V ; U H)$ and $(L V ; L H)$ are listed below each leaf image.

$r_{i}$ represents the radius of the incircle of the leaf,

$r_{c}$ the radius of the circumcircle of the leaf.

The ratio between the length of the main inertia axis and the minor inertia axis of the leaf, determines the accent of the leaf. It evaluates how much an iconic section deviates from being circular,

$$
E=\frac{E_{A}}{E_{B}} .
$$

The circularity $(C)$ is defined by all the contour points of the leaf image,

$$
C=\frac{\mu_{R}}{\sigma_{R}}
$$

where

$$
\mu_{R}=\frac{1}{N} \sum_{i=0}^{N-1}\left\|\left(x_{i}, y_{i}\right)-(\bar{x}, \bar{y})\right\|
$$

and

$$
\sigma_{R}=\frac{1}{N} \sum_{i=0}^{N-1}\left(\left\|\left(x_{i}, y_{i}\right)-(\bar{x}, \bar{y})\right\|-\mu_{R}\right)^{2}
$$

\section{CLASSIFIERS}

In the classification process, two well-known pattern classifiers will be used, MLP and RBF. They have been used to solve problems such as pattern classification and the approximation of functions. There are strengths and weaknesses associated to each classifier. Despite the high computational cost of the MLP and the sensitivity to the over fitting problem, the MLP has the ability to detect complex nonlinear relations between related and non-related variables. The RBF is very easy to design, the capabilities are very good and it performed robustly even when there are noises on the input. Depending on the problem each classifier will perform differently. In all the experiments the MLP configuration will depend on the number of inputs. For the first two experiments the input layer will have 4 or 8 neurons based on the number of input features. 100 neurons were used for the output layer because there are 100 species. There will be 2 hidden layers of 100 neurons each. The function used in the neurons in the output and hidden layer was the hyperbolic tangent function. To take advantage of the differentiability and non-linearity properties. In addition the following parameters are used: 500 training epochs with a learning rate of 0.1 , minimum performance gradient of $10^{-6}$, a maximum training time of $120 \mathrm{~s}$, a validation check of 500 and a performance goal of 0 . In the case of RBF the configuration is based on a Mean Square Error (MSE) goal of 0 , spread of $0.1,4$ and also 8 neurons in the input layer for the first experiment. The proposed RBF classifier contains one hidden layer on which some neurons are added until it meets the specified mean square error goal. The training step stop when 400 neurons are reached on the hidden layer, as shown by Adetiba and Olugbara (2015). Both classifiers were implemented on Matlab using Matlab's machine learning packages. For the classification phase $1 / 2$ of the data set were used for training and $1 / 4$ for testing and the rest for validation.

\section{RESULTS}

For each selected leaf, the associated grey scale image will be used during the feature extraction process. A sorted vector of sinuosity measure of each curve composing the leaf shape was extracted (sinuosity coefficients), followed by the extraction of the geometrical features and the 4 Fourier descriptors. For the first, second, third and fourth experiment the geometrical features ( 4 features), 4 sinuosity (4 features), 8 sinuosity ( 8 features) and 4 Fourier descriptors ( 4 features) and 8 Fourier descriptors $(8$ 
features) are respectively used to recognized leaf images. On the fifth, sixth, seventh, height and ninth experiments, the 4 sinuosity, 8 sinuosity and 4 Fourier descriptors and 8 Fourier descriptors are combined to the geometrical features to recognized leaf images. A total of $8,12,8$ and 12 features are respectively used for the classification.

The choice of four Fourier descriptors is because the sinuosity measure also used four values. The experimentation is performed using all the species in LeafSnap database.

Table 1 presents the classification results when using geometrical features, 4 sinuosity, 8 sinuosity and 4 Fourier descriptors as leaf features on the LeafSnap dataset. A classification rate of $70 \%$ was obtained using the MLP (Multilayer Perceptron) and $80 \%$ when using the RBF with the geometrical features as input. A classification rate of $80 \%$ was obtained with MLP and $92 \%$ with the RBF when using the 8 sinuosity coefficients.

Table 1 also presents the results of the classification when using the geometric features combined respectively to the 4 sinuosity coefficients, 8 sinuosity coefficients and to the Elliptic Fourier descriptors to characterize the leaves on the selected dataset. A classification rate of $82 \%$ was obtained with MLP when using the combination of geometrical features and 8 sinuosity coefficients and 93\% with the RBF on the database. Finally a classification rate of $80 \%$ was obtained with MLP and $91 \%$ with RBF respectively, using the 4 Elliptic Fourier descriptors combined to the geometric features. The Elliptic Fourier descriptors are obtained using a method describe by Kuhl and Giardina (1982).

\section{DISCUSSION}

The classification results of the geometric features with MLP are shown in the third row, second column and third column of Table 1. A classification rate of $70 \%$ is obtained, with a Mean Square Error (MSE) of 0.0160 . A classification rate of $80 \%$ with an MSE of 0.015 was also obtained when the geometrical features were used to characterize the shape of a leaf image with RBF as a classifier. These results show that the boundary shape features area able to recognized more than half of the leaf image. The results of the sinuosity coefficients and MLP classifier are shown in the third row, column four and five of Table 1 . A classification rate of $65 \%$ was obtained, with an MSE of 0.0274 with the MLP classifier. A classification rate of $88 \%$ with an MSE of 0.018 was also obtained with RBF classifier. These results show that the 4 sinuosity coefficients capture the leaf shape, structure but not with a high precision. That precision is improved when using the 8 sinuosity coefficients

The classification results when the 4 sinuosity coefficients are combined with geometrical features using MLP as the classifier are shown in the third row and column two of Table 1. A classification rate of $76 \%$ and a MSE of 0.015 were obtained. A classification rate of $90 \%$ and a MSE of 0.0047 were also obtained with the RBF classifier when using the 4 Sinuosity Coefficients combined with the geometrical features to characterize the shape of the leaf images. The results in Table 1 show that 4 sinuosity coefficients combined with the geometric features are more efficient than the 4 sinuosity coefficients.

From the results obtained in this study and the values observed on the subset of the confusion matrix in Table 2, it is shown that the sinuosity coefficients are good descriptors of leaf shape. However, further classification improvement was obtained by combining the 8 Sinuosity Coefficients with geometrical features compared to the combination of the geometrical features to the 4 elliptic Fourier descriptors.

The MLP performed poorly compared to the RBF on the overall dataset. The lower results observed are due to the fact that some leaves look alike and produce similar feature values. The superior performance of the RBF over MLP has also been reported in the literature, as presented by Adetiba and Olugbara (2015).

\section{CONCLUSIONS}

This paper has presented a model for leaf shape analysis based on the sinuosity coefficients and geometrical features. The proposed features for leaf shape characterization are translation and scale invariant. The experiments show that the sinuosity coefficients combined with geometrical features are efficient descriptors of leaf shape. A classification rate of $93 \%$ was achieved using the combination of Geometric features and 8 sinuosity coefficients with RBF as classifier on LeafSnap dataset. The optimal number of Sinuosity Coefficients used to describe a given shape is 8 , this constitute one of the limitation of the Sinuosity Coefficients and the fact that the rotation invariance remain an issue to investigate. Further analysis of the sinuosity coefficient for the characterization of the variations on leaf edges, and the demonstration of the rotation invariance of the sinuosity coefficients is envisioned. 
Table 1. Performance evaluation of all the features.

\begin{tabular}{|c|c|c|c|c|c|c|c|c|c|c|}
\hline & \multicolumn{2}{|c|}{ Geometric Features } & \multicolumn{2}{|c|}{ Sinuosity Coefficients } & \multicolumn{2}{|c|}{8 Sinuosity Coefficients } & \multicolumn{2}{|c|}{4 Fourier Descriptors } & \multicolumn{2}{|c|}{8 Fourier Descriptors } \\
\hline & MLP & $\mathrm{RBF}$ & MLP & $\mathrm{RBF}$ & MLP & RBF & MLP & RBF & MLP & RBF \\
\hline Sensitivity & 0.91 & 0.92 & 0.84 & 0.96 & 0.894 & 0.98 & 0.860 & 0.878 & 0.90 & 0.95 \\
\hline Specificity & 0.921 & 0.93 & 0.94 & 0.98 & 0.974 & 0.985 & 0.950 & 0.965 & 0.97 & 0.98 \\
\hline \multirow[t]{7}{*}{ Classification Rate } & 0.7 & 0.8 & 0.65 & 0.88 & 0.8 & 0.92 & 0.78 & 0.89 & 0.90 & 0.95 \\
\hline & & & \multicolumn{2}{|c|}{4 Sinuosity Coefficients } & \multicolumn{2}{|c|}{8 Sinuosity Coefficients } & \multicolumn{2}{|c|}{4 Fourier Descriptors } & \multicolumn{2}{|c|}{8 Fourier Descriptors } \\
\hline & & & \multicolumn{2}{|c|}{ Geometric Features } & \multicolumn{2}{|c|}{ Geometric Features } & \multicolumn{2}{|c|}{ Geometric Features } & \multicolumn{2}{|c|}{ Geometric Features } \\
\hline & & & MLP & $\mathrm{RBF}$ & MLP & RBF & MLP & RBF & MLP & $\mathrm{RBF}$ \\
\hline & & & 0.82 & 0.92 & 0.810 & 0.916 & 0.83 & 0.901 & 0.921 & 0.930 \\
\hline & & & 0.916 & 0.967 & 0.955 & 0.987 & 0.947 & 0.970 & 0.955 & 0.98 \\
\hline & & & 0.76 & 0.92 & 0.82 & 0.93 & 0.8 & 0.91 & 0.88 & 0.94 \\
\hline
\end{tabular}

Table 2. Subset of the confusion matrix.

\begin{tabular}{|c|c|c|c|c|c|c|c|c|c|}
\hline & Species 1 & Species 2 & Species 3 & Species 4 & Species 5 & Species 6 & Species 7 & Species 8 & Species 9 \\
\hline Species 1 & 11 & 3 & 0 & 0 & 0 & 0 & 0 & 0 & 0 \\
\hline Species 2 & 2 & 16 & 0 & 0 & 0 & 0 & 0 & 0 & 0 \\
\hline Species 3 & 0 & 0 & 18 & 0 & 0 & 0 & 0 & 0 & 0 \\
\hline Species 4 & 0 & 0 & 0 & 17 & 1 & 0 & 0 & 0 & 0 \\
\hline Species 5 & 0 & 0 & 0 & 0 & 17 & 0 & 0 & 0 & 0 \\
\hline Species 6 & 0 & 0 & 0 & 0 & 0 & 18 & 0 & 0 & 0 \\
\hline Species 7 & 0 & 0 & 0 & 0 & 0 & 0 & 14 & 0 & 0 \\
\hline Species 8 & 0 & 0 & 2 & 0 & 0 & 0 & 0 & 13 & 0 \\
\hline Species 9 & 0 & 0 & 0 & 0 & 0 & 0 & 0 & 0 & 17 \\
\hline
\end{tabular}

\section{REFERENCES}

Adetiba E, Olugbara OO (2015). Improved Classification of Lung Cancer Using Radial Basis Function Neural Network with Affine Transforms of Voss Representation. PloS One 10(12):e0143542.

Aickelin U, Bentley P, Cayzer S, Kim J, McLeod J (2003). Danger theory: The link between AIS and IDS? In: Timmis J, Bentley PJ, Hart E, eds. Artificial Immune Systems. Lect Not Comput Sci 2787:147-55.

Bendiab E, Kholladi MK (2011). Recognition of plant leaves using the dendritic cell algorithm. Int J Digi Inf Wirel Comm 1(1):284-92.

Cerutti G, Tougne L, Coquin D, Vacavant A (2013). Curvature-scale-based contour understanding for leaf margin shape recognition and species identification. In: Proc Int Conf Comput Vision Theo Appl (VISAPP).
Barcelona, Spain 1:277-84.

Cerutti G, Tougne L, Coquin D, Vacavant A (2014). Leaf margins as sequences: A structural approach to leaf identification. Pattern Recogn Lett 49:177-84.

Chaudhuri D, Samal A (2007). A simple method for fitting of bounding rectangle to closed regions. Pattern Recogn 40(7):1981-9.

Cope JS, Corney D, Clark JY, Remagnino P, Wilkin $P$ (2012). Plant species identification using digital morphometrics: A review. Expert Syst Appl 39(8):7562-73.

Fu H, Chi Z, Feng D, Song J (2004). Machine learning techniques for ontology-based leaf classification. In: Proc 8th Int Control Autom Robot Vision Conf (ICARCV). Kunming, China 1:681-6. 
Gonzalez RC, Woods RE (1992). Digital image processing. Reading: Addison-Wesley.

Jaekel M, Wake DB (2007). Developmental processes underlying the evolution of a derived foot morphology in salamanders. Proc Natl Acad Sci USA 104(51):20437-42.

Kala JR, Viriri S, Tapamo JR (2014). An approximation based algorithm for minimum bounding rectangle computation. In: Proc 6th IEEE Int Conf Adapt Sci Tech (ICAST). Ota, Nigeria 1-6.

Kala JR, Viriri S, Moodley D (2016). Sinuosity coefficients for leaf shape characterisation. In: Pillay N, Engelbrecht A, Abraham A, du Plessis M, Snášel V, Muda A, eds. Advances in nature and biologically inspired computing. Adv Intel Syst Comput 419:141-50.

Kalyoncu C, Toygar Ö (2015). Geometric leaf classification. Comput Vis Image Und 133:102-9.

Kuhl FP, Giardina CR (1982). Elliptic Fourier features of a closed contour. Comput Vision Graph 18(3):236-58.

Kumar N, Belhumeur PN, Biswas A, Jacobs DW, Kress WJ, Lopez IC, Soares JV (2012). Leafsnap: A computer vision system for automatic plant species identification. In Fitzgibbon A, Lazebnik S, Perona P, Sato Y, Schmid C, eds. Computer Vision - ECCV 2012. Lect Not Comput Sci 7573:502-16.

Langbein WB, Leopold LB (1966). River meanders-theory of minimum variance, USGS Prof. Paper 422-H. 15:1966.

Lazarus ED, Constantine JA (2013). Generic theory for channel sinuosity. Proc Natl Acad Sci USA 110(21):8447-52.
Lei YK, Zou JW, Dong T, You ZH, Yuan Y, Hu Y (2014). Orthogonal locally discriminant spline embedding for plant leaf recognition. Comput Vis Image Und 119:11626.

von Linné, Carl (1788). Systema naturæ per regna tria naturæ secundum classes, ordines, genera, species,... Vol. 1. Impensis Georg. Emanuel Beer.

Mallah C, Cope J, Orwell J (2013). Plant leaf classification using probabilistic integration of shape, texture and margin features. Signal Proc Pattern Recogn Appl 27986.

Naing L, Winn T, Rusli BN (2006). Practical issues in calculating the sample size for prevalence studies. Arch Orofac Sci 1(1):9-14.

Partington CF (1837). The British cyclopædia of natural history: Combining a scientific classification of animals, plants, and minerals. Orr \& Smith; 1837.

Tanguy A, Peuchot B, eds (2002). Research into spinal deformities 3. Amsterdam: IOS Press.

Wilf P, Zhang S, Chikkerur S, Little SA, Wing SL, Serre T (2016). Computer vision crack the leaf code. Proc Natl Acad Sci USA 113(12):3305-10.

Wu SG, Bao FS, Xu EY, Wang YX, Chang YF, Xiang QL (2007). A leaf recognition algorithm for plant classification using probabilistic neural network. In: Signal Processing and Information Technology, 2007 IEEE International Symposium on 2007 Dec 15 (pp. 1116). IEEE.

Yanikoglu B, Aptoula E, Tirkaz C (2014). Automatic plant identification from photographs. Machine Vision Appl 25(6):1369-83. 